\title{
Intermittent nature of solar wind turbulence near the Earth's bow shock: Phase coherence and non-Gaussianity
}

\author{
D. Koga, ${ }^{*}$ A. C.-L. Chian, and R. A. Miranda ${ }^{\dagger}$ \\ National Institute for Space Research (INPE) and World Institute for Space Environment Research (WISER), P.O. Box 515, \\ São José dos Campos, São Paulo, 12227-010, Brazil \\ E. L. Rempel \\ Institute of Aeronautical Technology (ITA) and World Institute for Space Environment Research (WISER), CTA/ITA/IEFM, \\ São José dos Campos, São Paulo, 12228-900, Brazil \\ (Received 10 November 2006; published 16 April 2007)
}

\begin{abstract}
The link between phase coherence and non-Gaussian statistics is investigated using magnetic field data observed in the solar wind turbulence near the Earth's bow shock. The phase coherence index $C_{\phi}$, which characterizes the degree of phase correlation (i.e., nonlinear wave-wave interactions) among scales, displays a behavior similar to kurtosis and reflects a departure from Gaussianity in the probability density functions of magnetic field fluctuations. This demonstrates that nonlinear interactions among scales are the origin of intermittency in the magnetic field turbulence.
\end{abstract}

DOI: 10.1103/PhysRevE.75.046401

PACS number(s): 52.35.- g, 95.30.Qd, 47.27.- $-\mathrm{i}$

Solar wind is a good laboratory for the study of collisionless magnetohydrodynamic (MHD) turbulence (see, e.g., [1] and references therein). In particular, the intermittent nature of turbulence is one of the fundamental problems for understanding the complex behavior of fluids [2,3] and other dynamical systems [4]. Solar wind intermittency can be characterized by the probability density functions (PDFs) of velocity (or magnetic) field fluctuations over a range of scales. For large scales the PDFs are approximately Gaussian. As the scale decreases, the tails of the distribution gradually become fatter [5].

Since MHD turbulence is governed by nonlinear MHD equations, the turbulent fields may display non-Gaussian fluctuations where the phases among scales (e.g., phases of the Fourier modes) are not random. In some previous studies of MHD turbulence the so-called random-phase approximation has been adopted to describe random-phase mixing among scales [6]. However, in solar wind turbulence coherent structures such as solitonlike waves are often observed, especially near the planetary bow shock [7]. Therefore, in real situations, the description of MHD turbulence as a superposition of random-phase fluctuations may not be valid and a finite-phase correlation among scales is to be expected due to nonlinear wave-wave interactions. This paper investigates the link between non-Gaussianity (intermittency) and phase correlation (nonlinear interactions) among scales in solar wind turbulence. Previous works have revealed the nonGaussianity of PDFs in the solar wind as a signature of intermittency, but whether this departure from Gaussianity is due to nonlinear wave-wave interactions or not has not been clearly demonstrated yet. In analytic modeling and numerical simulations of intermittent turbulence based on a set of deterministic equations, it is naturally expected that the depar-

\footnotetext{
*Electronic address: daiki@dge.inpe.br

${ }^{\dagger}$ Also at Centre for Quaternary Research (CEQua), University of Magallanes, P.O. Box 113-D, Punta Arenas, Chile.
}

ture from Gaussianity is due to nonlinear interactions [2]. In contrast, the observational data from solar wind are an admixture of deterministic signal and stochastic noise. In such a case, the demonstration of finite phase coherence is required to ascertain the nonlinear origin of non-Gaussian fluctuations. In the present work, we quantify the degree of nonlinear interactions in solar wind data using a phase coherence index and demonstrate its relation with kurtosis (flatness) in the structure function.

A central assumption of the Kolmogorov 1941 (hereafter $\mathrm{K} 41$ ) theory is the self-similarity of the random velocity field at inertial-range scales. This is related to the absence of phase correlation among scales. Since such random-phase fluctuations have self-similar hierarchical structures, the length of fluctuations, which is related to fractal dimension [8], will be long. In this paper, the length of discretized magnetic field fluctuations is defined using the Euclidean distance $S=\sum_{i=1}^{N} \sqrt{\left(B_{i+\tau}-B_{i}\right)^{2}+\left(t_{i+\tau}-t_{i}\right)^{2}}$, where $B$ and $t$ correspond to the discretized magnetic field fluctuations (the measured time series) and time, respectively, $\tau$ denotes the time lag between two points in the time series, and $N$ is the number of data points. On the other hand, if there exists finite phase correlation among scales due to nonlinear wavewave interactions, then the length of phase-correlated fluctuations becomes shorter than the random-phase fluctuations because of the increase of two-point correlations. Thus, the degree of phase correlation among scales can be evaluated in terms of $S$. To this end, we apply the technique of phase coherence (PC) index $C_{\phi}(\tau)$ [9]. From the original magnetic field data (ORG), we make two surrogate data. First we decompose the observational data into the power spectrum and the phases by using the Fourier transform. Next we randomly shuffle the phases, but keep the power spectrum unchanged, and from these two sets of information in Fourier space, we perform the inverse Fourier transform to create the phaserandomized surrogate (PRS) [10]. Likewise we can make the phase-correlated surrogate (PCS), in which the phase differences are all made equal. The three data sets ORG, PRS, and 
PCS share exactly the same power spectrum, while their phase distributions are all different. Each length for ORG, PRS, and PCS data is measured by a simple two-point difference $S_{1}(\tau)=\sum_{i=1}^{N}\left|B_{i+\tau}-B_{i}\right|$, instead of the Euclidean distance to reduce the computational time. Then the PC index is defined as follows:

$$
C_{\phi}(\tau)=\frac{S_{P R S}(\tau)-S_{O R G}(\tau)}{S_{P R S}(\tau)-S_{P C S}(\tau)} .
$$

The calculation of $S_{P R S}(\tau)$ is performed using an average over 100 realizations of the phase shuffling. If the phases among scales for the original data are completely random, then $C_{\phi}(\tau)=0$, while $C_{\phi}(\tau)=1$ if the phases are completely correlated.

Furthermore, higher-order statistics is also useful to characterize the intermittent nature of fluctuations when the PDFs depart from a Gaussian distribution. In particular, the fourth-order moment (kurtosis)

$$
K(\tau)=\frac{1}{N} \sum_{i=1}^{N}\left(\frac{u_{i}-\left\langle u_{i}\right\rangle}{\sigma}\right)^{4}-3
$$

is an important quantity to evaluate the degree of intermittency, where the brackets $\langle\cdot\rangle$ denote the ensemble average of fluctuation $u_{i}=B_{i+\tau}-B_{i}$ and $\sigma$ indicates its standard deviation. If $K(\tau)$ is zero, the PDFs are Gaussian distributions, while if $K(\tau)>0$, the PDFs show a sharp peak around its mean and fat tails.

We analyze two time series of the total magnetic field data, $B$, observed upstream and downstream of the Earth's bow shock by the GEOTAIL satellite, sampled at $16 \mathrm{~Hz}$ (from 18:00 UT 8 October to 04:00 UT 9 October 1995). Since this data were observed in the declining phase of the solar cycle 22 and did not include any special events with high-speed flows and coherent structures originating from the Sun-e.g., coronal mass ejection [12]—it can be treated as a moderate state of MHD turbulence near the Earth's bow shock. In order to separate the data into upstream and downstream regions, the velocity and density data are used with the bow shock model introduced by Fairfield [11]. Each time series has 16384 samples, which is appropriate for evaluation of higher-order moments (at least up to sixth order) [13]. Slow trends are subtracted from the original data using a quadratic function. In Fig. 1, the scale dependence of the magnetic field fluctuations $\delta B_{i}=B_{i+\tau}-B_{i}$ for three different scales $(\tau=1,4$, and $16 \mathrm{~s}$ ) is shown for the upstream (top panel) and downstream (bottom panel) time series. For both upstream and downstream, as the scale becomes smaller, the fluctuations become more intermittent.

The intermittent characteristics of the fluctuations $\delta B$ can be visualized in their PDFs. The fluctuations are normalized as $\delta b=(\delta B-\langle\delta B\rangle) / \sigma$, where the brackets denote the ensemble average of $\delta B$ and $\sigma$ denotes the standard deviation. Figure 2 presents the PDFs for the upstream (left column) and downstream (right column) fluctuations for $\tau=1,4$, and $16 \mathrm{~s}$ (circles), superposed by a Gaussian PDF (solid line). For both upstream and downstream fluctuations, as the scale $\tau$ decreases, the PDF deviates from the Gaussian distribution.
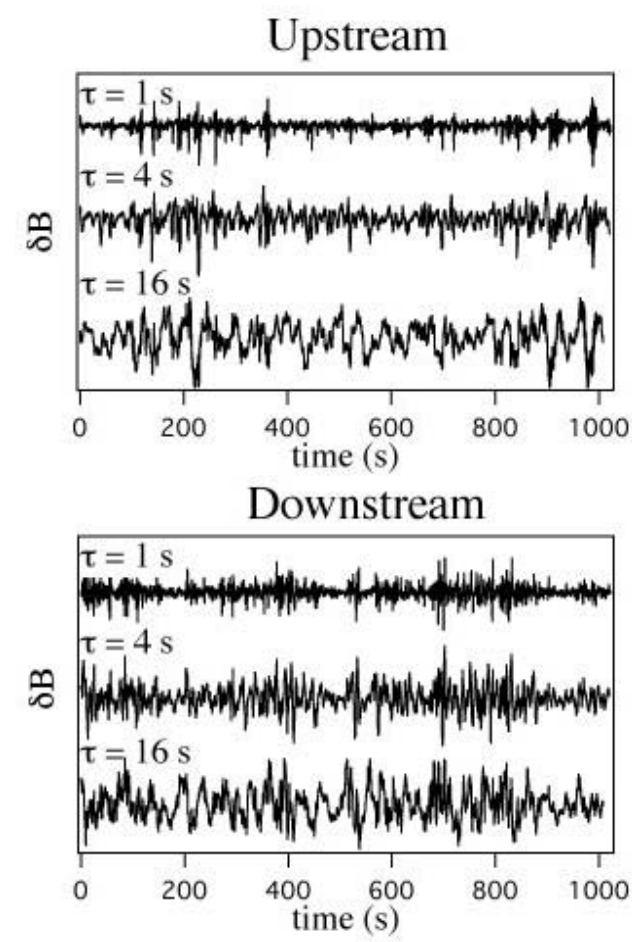

FIG. 1. Scale dependence of the magnetic field fluctuations $\delta B(t)=B(t+\tau)-B(t)$ near the Earth's bow shock for three different scales $(\tau=1,4$, and $16 \mathrm{~s})$. The top panel corresponds to the upstream case, and the bottom panel corresponds to the downstream case.

Thus, the smaller the scale, the shorter the interspike interval, implying stronger intermittency. This is in agreement with previous works on the behavior of PDFs obtained from solar wind turbulent fluctuations $[1,5]$. For the upstream fluc-

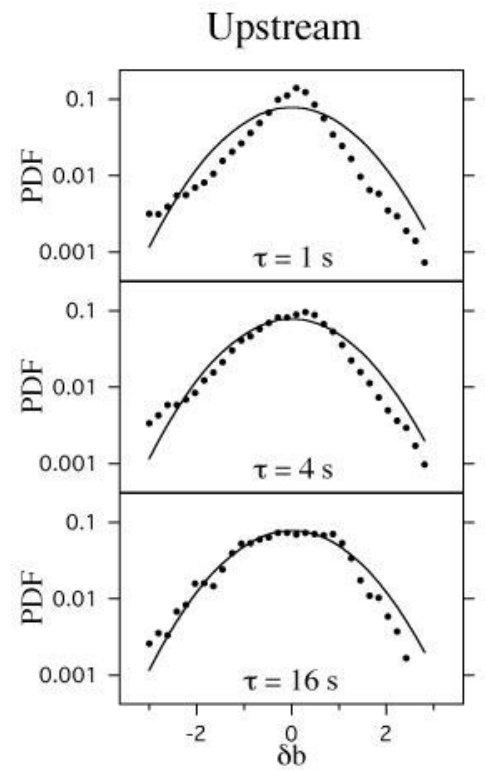

\section{Downstream}

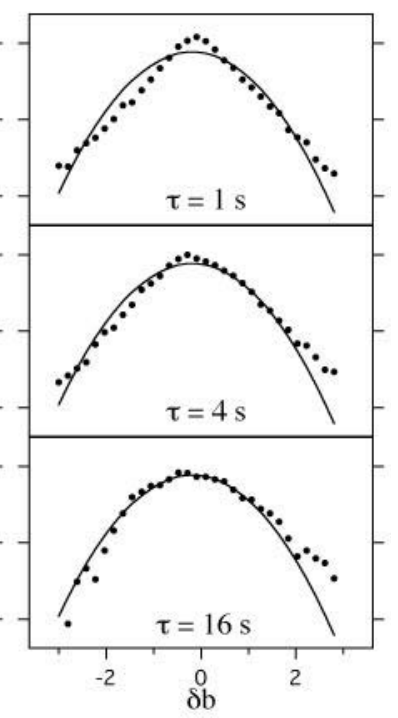

FIG. 2. The scaling behavior of the PDFs for the magnetic field fluctuations in the upstream region (left) and the downstream region (right) of the Earth's bow shock. The circles denote the PDFs for magnetic field fluctuations. The solid lines denote Gaussian PDFs. 


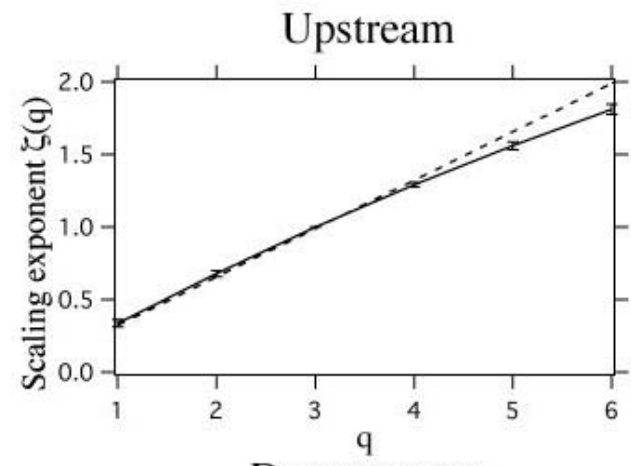

Downstream

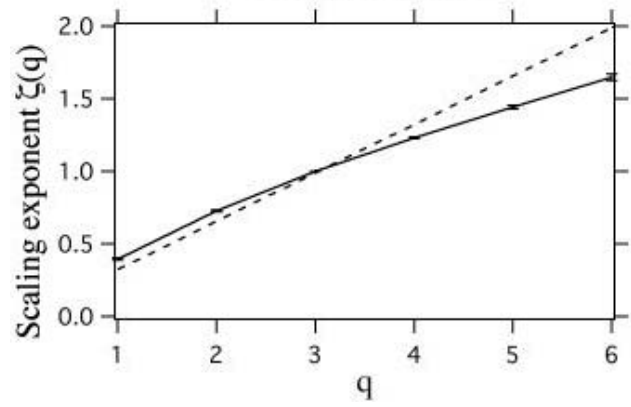

FIG. 3. Scaling exponent $\zeta_{q}$ of the $q$ th-order structure function obtained by ESS fitting for the upstream and downstream data sets. The dashed line corresponds to $\mathrm{K} 41\left(\zeta_{q}=q / 3\right)$ linear scaling. The error of the least-squares fitting is indicated by the bars.

tuations, from $\tau=16 \mathrm{~s}$ to $\tau=1 \mathrm{~s}$, the behavior of the PDFs is similar to that of the downstream data except for its asymmetry. This indicates that the dynamics in the upstream and downstream regions of the Earth's bow shock is quite different. The fat tail for the negative fluctuations of the upstream PDF at $\tau=1 \mathrm{~s}$ suggests that low-frequency large-amplitude MHD waves being convected toward the Earth steepen with time as the satellite approaches the bow shock. This fact has been widely discussed in the literature. For example, the steepened waves, shocklets, and short large-amplitude mag- netic structures (SLAMSs) often observed upstream of the Earth's bow shock are believed to result from a nonlinear evolution of low-frequency large-amplitude MHD waves as the solar wind convects towards the Earth's bow shock [14]. For the downstream case, such steepening has not been observed.

In order to quantify the deviation from a Gaussian distribution, the Kolmogorov universality theory is used. To this end, the structure function for each moment, $S_{q}(\tau)$ $=\left\langle\left|B_{i+\tau}-B_{i}\right|^{q}\right\rangle \sim \tau^{\alpha(q)}$, is normalized with respect to the third-order structure function-i.e., extended self-similarity (ESS) [15]. Figure 3 shows the scaling exponent of the normalized $q$ th-order structure function $S_{q}(\tau) \sim\left[S_{3}(\tau)\right]^{\zeta(q)}$ where $\zeta(q) \simeq \alpha(q) / \alpha(3)$ and the dashed line stands for the K41 scaling, $\alpha(q)=q / 3$. For both cases, a deviation from the K41 scaling is clearly shown in the figure. In agreement with Fig. 2 , the deviation from the Gaussian curve is due to the increase of the rate of occurrence of small- and largeamplitude fluctuations in both regions.

The results from Figs. 1 and 2 indicate that both data sets consist of an admixture of Gaussian (large-scale) and nonGaussian (inertial range) fluctuations and display multifractal features. The non-Gaussianity can be related to phase coherence by computing the PC index. The profile of PC index for the upstream region is shown as a function of $\tau$ in the bottom left panel of Fig. 4. The PC index increases monotonically from $\tau \sim 16 \mathrm{~s}$ (c) to $\tau \sim 1 \mathrm{~s}$ (a) while it decreases for $\tau \lesssim 1 \mathrm{~s}$. The range in which the PC index increases corresponds, approximately, to a frequency range of from $\sim 0.1 \Omega_{i}$ to $\sim \Omega_{i}$, where $\Omega_{i}$ denotes the local ion-cyclotron frequency in the upstream region (approximately $1 \mathrm{~Hz}$ ) [9]. In the upstream region, low-frequency MHD waves are excited mainly by ion-beam instabilities. Once they reach finite amplitudes, they can evolve nonlinearly in the presence of inhomogeneous spatial distributions of plasma. Such a nonlinear evolution of MHD waves is closely related to the appearance of a high-PC index region since the phase coherence among scales is a manifestation of nonlinear interactions. The decrease of the PC index for $\tau \lesssim 1 \mathrm{~s}$ implies that

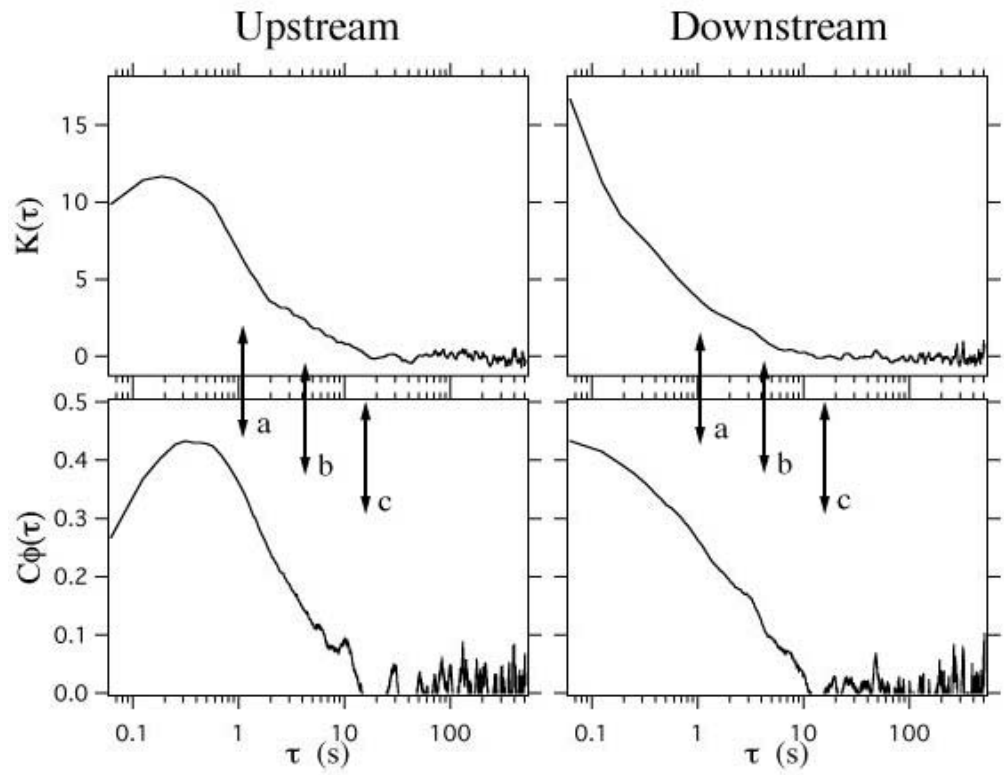

FIG. 4. Kurtosis (top panel) and PC index (bottom panel) for the upstream and downstream regions of the Earth's bow shock. The arrows (a), (b), and (c) correspond to scales $\tau=1,4$, and $16 \mathrm{~s}$, respectively. 
nonlinear interactions among scales less than $1 \mathrm{~s}$ become weak due to energy dissipation processes such as Landau damping. The top left panel in Fig. 4 shows the value of kurtosis. There is a significant deviation from zero (Gaussianity) around $\tau \sim 1 \mathrm{~s}$. This result is consistent with the value of PC index. For $\tau \gtrsim 10 \mathrm{~s}$, the PC index fluctuates around 0.1 , while the value of kurtosis is also small. This indicates that for large scales the nonlinear interactions are weak and magnetic fluctuations are almost stochastic.

Recalling the results shown in Fig. 2, the variation of the PC index clearly corresponds to that of PDFs. In the downstream case, the PC index increases monotonically from $\sim 16 \mathrm{~s}$ (c) to the smallest scale in the bottom right panel of Fig. 4. This result indicates that in the downstream region the range of nonlinear wave-wave interactions (i.e., inertial range) extends to scales smaller than the upstream region. The value of kurtosis is shown in the top right panel of Fig. 4. One can see that the tendency of kurtosis is in good agreement with that of the PC index. It has been reported that for MHD scales, Alfvén ion-cyclotron modes or mirror modes are dominant in the downstream region due to the ion temperature anisotropy [16]. The nonlinear evolution of such modes may be responsible for the phase coherence in the downstream MHD turbulence.

Recall that the definition of the length $S_{1}$ corresponds to the definition of the first-order structure function. Furthermore, for the PC index, we calculate the difference of the length between the original data and the phase randomized data (Gaussian distribution). The structure function is also defined as $S_{q}(\tau)=\int u^{q} P(u) d u$, where $u$ denotes the two-point difference of fluctuations (absolute value) and $P(u)$ indicates the probability density of $u$. Thus, the PC index indicates how much the PDFs deviate from the Gaussian distribution with the same mean and same variance as the original data. On the other hand, kurtosis is related to the fourth-order structure function, which also characterizes the departure from Gaussian distribution (excess of large-amplitude fluctuations). Therefore, the similarity between the PC index using the definition of first-order structure function and kurtosis can be naturally noticed.

In this study we established the relation between nonGaussianity of PDFs and phase coherence in intermittent time series, based on the observational data of magnetic field fluctuations near the Earth's bow shock. We found that the behavior of the phase coherence index is similar to that of kurtosis, which demonstrates that intermittency is produced by the phase correlation among scales due to nonlinear wave-wave interactions. Although we only analyzed one set of data in this study, we have performed a more exhaustive data analysis of the approach described in this paper using various data sets for both weak and strong states of MHD turbulence in space as well as for atmospheric turbulence. In addition, we have verified that the same technique is valid for the intermittent time series obtained from the numerical solutions of several nonlinear wave equations. These results will be published shortly. Hence, we are confident that our technique is robust and applicable to nonlinear data in general.

This research is supported by FAPESP(04/15942-2) and $\mathrm{CNPq}$, Brazil. The authors thank the investigators of the GEOTAIL magnetic field (MGF), Professor S. Kokubun and Professor T. Nagai, for providing the data.
[1] R. Bruno and V. Carbone Living Rev. Solar Phys. 2, 4 (2005).

[2] U. Frisch, Turbulence-The Legacy of A. N. Kolmogorov (Cambridge University Press, Cambridge, England, 1995).

[3] T. Chang, S. W. Y. Tam, and C. C. Wu, Phys. Plasmas 11, 1287 (2004); B. Hnat, S. C. Chapman, and G. Rowlands, Phys. Rev. E 67, 056404 (2003); B. Hnat, S. C. Chapman, and G. Rowlands, ibid. 72, 029902(E) (2005).

[4] A. C.-L. Chian, E. L. Rempel, E. E. Macau, R. R. Rosa, and F. Christiansen, Phys. Rev. E 65, 035203(R) (2002); K. He and Abraham C.-L. Chian, Phys. Rev. Lett. 91, 034102 (2003); K. $\mathrm{He}$ and Abraham C.-L. Chian, Phys. Rev. E 69, 026207 (2004); E. L. Rempel and Abraham C.-L. Chian, ibid. 71, 016203 (2005); E. L. Rempel and Abraham C.-L. Chian, Phys. Rev. Lett. 98, 014101 (2007).

[5] E. Marsch and C. Y. Tu, Ann. Geophys. 12, 1127 (1994); L. Sorriso-Valvo et al., Geophys. Res. Lett. 26, 1801 (1999).

[6] R. Z. Sagdeev and A. A. Galeev, Nonlinear Plasma Theory (W. A. Benjamin, New York, 1969).

[7] S. J. Schwartz and D. Burgess, Geophys. Res. Lett. 18, 373 (1991); K. Stasiewicz, P. K. Shukla, G. Gustafsson, S. Buchert, B. Lavraud, B. Thide, and Z. Klos, Phys. Rev. Lett. 90,
085002 (2003).

[8] T. Higuchi, Physica D 31, 277 (1988).

[9] T. Hada, D. Koga, and E. Yamamoto, Space Sci. Rev. 107, 463 (2003); D. Koga and T. Hada, ibid. 107, 495 (2003); D. Koga, A. C.-L. Chian, T. Hada, and E. L. Rempel, Philos. Trans. R. Soc. London, Ser. A (to be published).

[10] T. Schreiber and A. Schmitz, Physica D 142, 346 (2000).

[11] D. H. Fairfield, J. Geophys. Res. 76, 6700 (1971).

[12] B. C. Low, J. Geophys. Res. 106, 25141 (2001).

[13] T. Dudok De Wit, Phys. Rev. E 70, 055302(R) (2004).

[14] C. T. Russell, D. D. Childer, and P. J. Coleman, Jr., J. Geophys. Res. 76, 845 (1971); M. M. Hoppe and C. T. Russell, Nature (London) 287, 417 (1980); S. J. Steven et al., J. Geophys. Res. 97, 4209 (1992).

[15] R. Benzi, S. Ciliberto, R. Tripiccione, C. Baudet, F. Massaioli, and S. Succi, Phys. Rev. E 48, R29 (1993).

[16] O. Alexandrova et al., J. Geophys. Res. 109, A05207 (2004); Y. Narita and K.-H. Glassmeier, ibid. 110, A12215 (2005); F. Sahraoui, G. Belmont, L. Rezeau, N. Cornilleau-Wehrlin, J. L. Pincon, and A. Balogh, Phys. Rev. Lett. 96, 075002 (2006). 